\title{
ON ONE EXTENSION THEOREM DEALING WITH WEIGHTED ORLICZ-SLOBODETSKII SPACE. ANALYSIS ON LIPSCHITZ SUBGRAPH AND LIPSCHITZ DOMAIN
}

\author{
Raj Narayan Dhara And AgnieszKa KaŁAmajska
}

Abstract. Having a given weight $\rho(x)=\tau(\operatorname{dist}(x, \partial \Omega))$ defined on Lipschitz boundary domain $\Omega$ and an Orlicz function $\Psi$, we construct the subordinated weight $\omega(\cdot, \cdot)$ defined on $\partial \Omega \times \partial \Omega$ and extension operator $\operatorname{Ext}^{L}: \operatorname{Lip}(\partial \Omega) \mapsto \operatorname{Lip}(\bar{\Omega})$ form Lipschitz functions defined on $\partial \Omega$ to Lipschitz functions defined on $\bar{\Omega}$, independent of $\tau$ and $\Psi$, in such a way that $\operatorname{Ext}^{L}$ extends to the bounded operator from the subspace of weighted Orlicz-Slobodetskii space $Y_{\omega}^{\Psi, \Psi}(\partial \Omega)$ generated by Lipschitz functions and subordinated to the weight $\omega$ to Orlicz-Sobolev space $W_{\rho}^{1, \Psi}(\Omega)$. More detailed analysis on Lipschitz subgraph is also provided. Result is new in the unweighted Orlicz setting for general function $\Psi$ as well as in the weighted $L^{p}$ setting.

Mathematics subject classification (2010): Primary 46E35; Secondary 26D10.

Keywords and phrases: Orlicz spaces, weighted Orlicz-Slobodetskii spaces, weighted Orlicz-Sobolev spaces, extension theorem, trace embedding theorem.

\section{REFERENCES}

[1] H. Abels, M. Krbec, K. Schumacher, On the trace space of a Sobolev space with a radial weight, J. Funct. Spaces Appl. 6 (2008), no. 3, 259-276.

[2] N. Aronszajn, Boundary value of functions with finite Dirichlet integral, Techn. Report 14, University of Kansas 1955.

[3] J. M. BALL, Convexity conditions and existence theorems in nonlinear elasticity, Arch. Rat. Mech. Anal. 63 (1977), 337-403.

[4] A. BenkiRane, Approximations de type Hedberg dans les espaces $W^{m} L \log L(\Omega)$ et applications, Annales de la faculté des sciences de Toulouse $5^{e}$ série, tome 11, no. 2, (1990), p. 67-78.

[5] S-S. BYUn, F. YAO, S. ZHOU, Gradient estimates in Orlicz space for nonlinear elliptic equations, J. Funct. Anal. 255 (2008), no. 8, 1851-1873.

[6] A. Caruso, Two properties of norms in Orlicz spaces, Matematiche (Catania) 56 (1) (2001), 183 194.

[7] J. Chabrows ki, The Dirichlet Problem with $L^{2}$-Boundary Data for Elliptic Linear Equations, Lecture Notes in Math., vol. 1482, Springer-Verlag, Berlin, 1991.

[8] A. Cianchi, Some results in the theory of Orlicz spaces and applications to variational problems, Nonlinear analysis, function spaces and applications, vol. 6 (Prague, 1998), Acad. Sci. Czech Rep., Prague 1999, pp. 50-92.

[9] A. CiAnchI, Orlicz-Sobolev boundary trace embeddings, Math. Z. 266 (2) (2010), 431-449.

[10] A. CiAnChI, R. Kerman, L. Pick, Boundary trace inequalities and rearrangements, J. Anal. Math. 105 (2008), 241-265.

[11] R. N. DharA, Existence of the solution of linear degenerated PDEs of elliptic type in weighted Sobolev spaces with non-homogeneous boundary condition, preprint: https://www.mimuw. edu.pl/badania/preprinty/preprinty-imat/?LANG=en.

[12] R. N. Dhara, A. KaŁamajska, On one extension theorem dealing with weighted OrliczSlobodetskii space. Analysis on cube, Mathematical Inequalities \& Applications 18, no. 1 (2015), 61-89. 
[13] T. K. Donaldson, N. S. Trudinger, Orlicz-Sobolev spaces and imbedding theorems, J. Functional Analysis 8 (1971), 52-75.

[14] P. Drabek, A. Kufner, F. Nicolasi, Quasilinear Elliptic Equations with Degenerations and Singularities, Walter De Gruyter Inc, 1997.

[15] E. B. FABES, C. E. Kenig, R. P. SERAPIONI, The local regularity of solutions of degenerate elliptic equations, Comm. Partial Differential Equations 7 (1982), no. 1, 77-116.

[16] A. FougÉRES, Thesis (in French), University of Besançon, 1972.

[17] A. Fougéres, Théorèmes de trace et de prolongement dans les espaces de Sobolev et Sobolev-Orlicz, (in French) C. R. Acad. Sci. Paris Sér. A-B 274 (1972), A181-A184.

[18] M. Frazier, S. Roudenko, Traces and extensions of matrix-weighted Besov spaces, Bulletin of the London Mathematical Society, 40 (2) (2008), 181-192.

[19] E. Gagliardo, Caratterizzazioni delle tracce sulla frontiera relative ad alcune classi di funzioni in $n$ variabili, (Italian) Rend. Sem. Mat. Univ. Padova 271957 284-305.

[20] M. Garcia-Huidobro, A. Kufner, R. Manásevich, C. S. Yarur, Radial solutions for a quasilinear equation via Hardy inequalities, Adv. Differential Equations 6 (2001), no. 12, 1517-1540.

[21] J.-P. Gossez, Nonlinear elliptic boundary value problems for equations with rapidly (or slowly) increasing coefficients, Trans. Amer. Math. Soc. 190 (1974), 163-205.

[22] J.-P. Gossez, V. Mustonen, Variational inequalities in Orlicz-Sobolev spaces, Nonlinear Anal. 11 (3) (1987), 379-392.

[23] J.-P. GosseZ, Some approximation properties in Orlicz-Sobolev spaces, Studia Math. 74 (1982), no. $1,17-24$.

[24] D. HARos Ke, H-J. SChmeISSER, On trace spaces of function spaces with a radial weight: the atomic approach, Complex Var. Elliptic Equ. 55 (2010), no. 8-10, 875-896.

[25] J. Heinonen, T. Kilpelainen, O. Martio, Nonlinear Potential Theory of Degenerate Elliptic Equations, Oxford Mathematical Monographs. The Clarendon Press Oxford University Press, New York, 1993.

[26] H. Hudzik, Density of $C_{0}^{\infty}\left(R^{n}\right)$ in generalized Orlicz-Sobolev space $W_{M}^{k}\left(R^{n}\right)$, Funct. Approx. Comment. Math. 7 (1979), 15-21.

[27] D. Jerison, CARLos E. KenIG, The inhomogeneous Dirichlet problem in Lipschitz domains, Journal of Functional Analysis 130 (1995), no. 1, 161-219.

[28] A. Jonsson, H. Wallin, Function spaces on subsets of $R^{n}$, Math. Rep., $2,1984$.

[29] A. KaŁamajska, Pointwise multiplicative inequalities and Nirenberg type estimates in weighted Sobolev spaces, Studia Math. 108 (1994), no. 3, 275-290.

[30] A. KaŁamajska, M. KRbec, Traces of Orlicz-Sobolev functions under general growth restrictions, Math. Nachr. 286, no. 7 (2013), 730-742.

[31] A. KaŁamajska, M. Krbec, On solutions to heat equation with the initial condition in OrliczSlobodetskii space, Proc. Roy. Soc. Edinburgh Sect. A 144, no. 4 (2014), 787-807.

[32] A. Kąamajska, K. Pietruska-PaŁuba, Gagliardo-Nirenberg inequalities in weighted Orlicz spaces, Studia Math. 173 (1) (2006), 49-71.

[33] T. Kilpelainen, Smooth approximation in weighted Sobolev spaces, Comment. Math. Univ. Carolin. 38 (1997), no. 1, 29-35.

[34] D. KIM, Trace theorems for Sobolev-Slobodeckij spaces with or without weights, J. Funct. Spaces Appl. 5 (3) (2007), 243-268.

[35] K-H. Kim, N. V. KRYLov, On the Sobolev space theory of parabolic and elliptic equations in $C^{1}$ domains, SIAM J. Math. Anal. 36 (2) (2004) 618-642 (electronic).

[36] H. KiTA, On Hardy-Littlewood maximal functions in Orlicz spaces, Math. Nachr. 183 (1997), 135155.

[37] V. M. KokılašHVILI, On traces of functions with partial derivatives from Orlicz classes, Special issue dedicated to Władysław Orlicz on the occasion of his seventy-fifth birthday. Comment. Math. Special Issue 1 (1978), 183-189.

[38] M. A. Krasnoselskit, YA. B. Rutickit, Convex Functions and Orlicz Spaces, P. Noordhoff Ltd. Groningen 1961.

[39] L. D. Kudryavcev, Direct and inverse imbedding theorems. Applications to the solution of elliptic equations by variational methods, Trudy Mat. Inst. Steklov. 55 (1959), 182 pp. (in Russian).

[40] A. Kufner, Weighted Sobolev spaces, A Wiley-Interscience Publication, John Wiley \& Sons, New York, 1985. 
[41] A. Kufner, O. John, S. FučÍK, Function spaces. Monographs and Textbooks on Mechanics of Solids and Fluids; Mechanics: Analysis, Noordhoff International Publishing, Leyden; Academia, Prague, 1977.

[42] M.-Th. Lacroix, Espaces de traces des espaces de Sobolev-Orlicz, J. Math. Pures Appl. 53 (9) (1974), 439-458.

[43] M.-TH. LACROIX, Echelle d'espaces intermédiaires entre un espace de Sobolev-Orlicz et un espace d'Orlicz. Trace d'espaces de Sobolev-Orlicz avec poids, (in French) Journées d'Analyse Non Linéaire (Proc. Conf., Besançon, 1977), 121-139, Lecture Notes in Math., 665, Springer, Berlin, 1978.

[44] M. LisowsKi, Approximation in Orlicz-Slobodeckii space by functions in $C^{\infty}(\Omega)$, Fasc. Math. no. 29 (1999), 43-54.

[45] M. LisowsKi, Approximation by functions in $C_{0}^{\infty}(\Omega)$ in Orlicz-Sobolev spaces, Fasc. Math. no. 36 (2005), 73-82.

[46] P. I. Lizorkin, Boundary properties of functions from "weight” classes, Dokl. Akad. Nauk SSSR 132 (1960), 514-517 (in Russian); translated as Soviet Math. Dokl. 1 (1960), 589-593.

[47] J-L. Lions, Un théoreme de traces; applications, (in French) C. R. Acad. Sci. Paris 249 (1959) 2259-2261.

[48] S. V. Lotots Ky, Linear stochastic parabolic equations, degenerating on the boundary of a domain, Electron. J. Probab. 6 (24) (2001), 1-14.

[49] V. G. MAZ'YA, J. Rossmann, Schauder estimates for solutions to a mixed boundary value problem for the Stokes system in polyhedral domains, Math. Methods Appl. Sci. 29 (9) (2006), 965-1017.

[50] S. Mayboroda, M. Mitrea, Sharp estimates for Green potentials on non-smooth domains, Mathematical Research Letters 11 (2004), 481-492.

[51] K. Brewster, I. Mitrea, M. MitreA, Stein's extension operator on weighted Sobolev spaces on Lipschitz domains and applications to interpolation, Recent advances in harmonic analysis and partial differential equations, 13-38, Contemp. Math., 581, Amer. Math. Soc., Providence, RI, 2012.

[52] M. Mitrea, M. TAYlor, The Poisson problem in weighted Sobolev spaces on Lipschitz domains, Indiana Univ. Math. J. 55 (2006), no. 3, 1063-1089.

[53] M. Mitrea, M. Taylor, Potential theory on Lipschitz domains in Riemannian manifolds: SobolevBesov space results and the Poisson problem, J. Funct. Anal. 176 (2000), no. 1, 1-79.

[54] J. NEČAS, Direct methods in the theory of elliptic equations, Translated from the 1967 French original by Gerard Tronel and Alois Kufner. Editorial coordination and preface by Šárka Nečasová and a contribution by Christian G. Simader. Springer Monographs in Mathematics. Springer, Heidelberg, 2012.

[55] J. NEČAS, Sur une méthode pour résoudre les équations aux dérivés partielles du type elliptique, voisine de la variationnelle, (in French) Ann. Scuola Norm. Sup. Pisa 16 (3) (1962) 305-326.

[56] S. M. Nikols KiI, Properties of certain classes of functions of several variables on differentiable manifolds, (in Russian) Mat. Sb. N.S. 33 (75), no. 2 (1953), 261-326.

[57] A. NeKvinda, Characterization of traces of the weighted Sobolev space $W^{1, p}\left(\Omega, d_{M}^{\varepsilon}\right)$ on $M$ (English), Czechoslovak Mathematical Journal, 43, no. 4 (1993), 695-711.

[58] A. NeKVinda, L. Pick, Characterization of traces of the weighted Sobolev space $H^{1, p}\left(\Omega, d_{M}^{\varepsilon}\right)$ on $M$ (English summary), Funct. Approx. Comment. Math. 20 (1992), 143-151.)

[59] G. PALMIERI, The traces of functions in a class of Sobolev-Orlicz spaces with weight (Italian. English summary), Boll. Un. Mat. Ital. B 5, 18 (1981), no. 1, 87-117.

[60] L. PICK, W. Sickel, Several types of intermediate Besov-Orlicz spaces, Math. Nachr. 164 (1993), $141-165$.

[61] I. Piotrows KA, Traces on fractals of function spaces with Muckenhoupt weights, Functiones et Approximatio Commentarii Mathematici, 36 (1) (2006), 95-117.

[62] V. R. Portnov, Two embedding theorems for the space $L_{p, b}^{1}\left(\Omega \times \mathbf{R}_{+}\right)$and their applications, (in Russian) Dokl. Akad. Nauk SSSR 155 (1964), 761-764.

[63] M. M. Rao, Z. D. Ren, Theory of Orlicz spaces, M. Dekker, Inc. New York, 1991.

[64] L. N. SLOBODETS KII, Sobolev spaces of fractional order and their application to boundary problems for partial differential equations (in Russian), Uch. Zapisky Leningrad. Ped. Inst. im. A. I. Gercena 197 (1958), 54-112.

[65] I. SKRZYPCZAK, Hardy-type inequalities derived from p-harmonic problems, Nonlinear Anal. 93 (2013), 30-50. 
[66] E. M. STEIn, T. S. MURPhy, Harmonic analysis: real-variable methods, orthogonality, and oscillatory integrals, Vol. 3. Princeton University Press, 1993.

[67] H. TRIEBEL, Theory of Function Spaces, (Vol. 2, 1992), Birkhüser, Berlin, 1983.

[68] A. I. TyUlenev, Characterization of traces of weighted Sobolev spaces, Trudy MFTI 3 (1) (2011), $141-145$.

[69] A. I. TyUlEnEV, The problem of traces for sobolev spaces with Muckenhoupt-type weights, Mathematical Notes, 94 (5-6) (2013), 668-680.

[70] A. A. VAŠARIN, The boundary properties of functions having a finite Dirichlet integral with a weight, (in Russian) Dokl. Akad. Nauk SSSR (N.S.) 117 (1957) 742-744.

[71] S. V. USPENSKIǏ, Imbedding theorems for classes with weights (in Russian), Trudy Mat. Inst. Steklov. 60 (1961), 282-303.

[72] V. V. ZHIKov, On the density of smooth functions in Sobolev-Orlicz spaces, Zap. Nauchn. Sem. S.Peterburg. Otdel. Mat. Inst. Steklov. (POMI) 310 (2004), Kraev. Zadachi Mat. Fiz. i Smezh. Vopr. Teor. Funkts. 35 [34], 67-81, 226; translation in J. Math. Sci. (N. Y.) 132 (2006), no. 3, 285-294. 